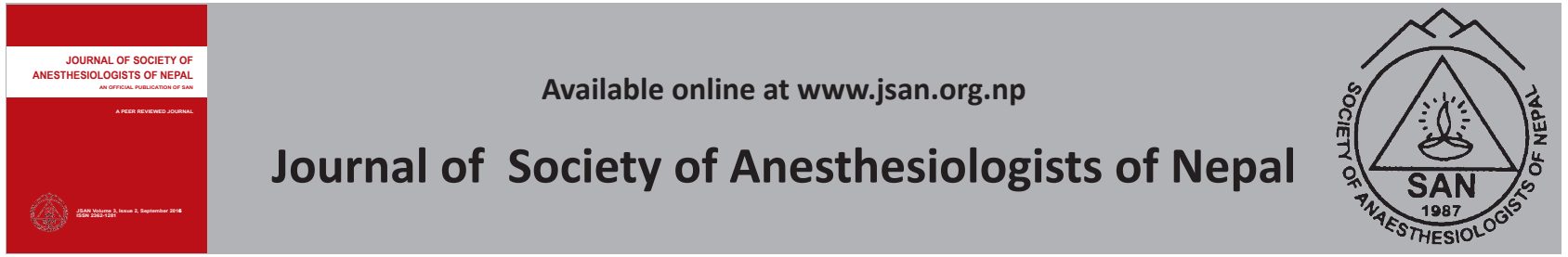

\title{
Editorial
}

\section{Guidelines for reporting health research: what authors should check before submitting an article}

\author{
Apurb Sharma ${ }^{*}$, Diptesh Aryal ${ }^{\ddagger}$
}

¥Shahid Gangalal National Heart Centre, Bansbari, Kathmandu 44606, Nepal

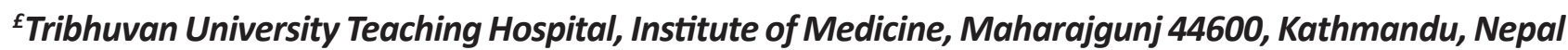

W th the publication of the 2016 September issue, Journal of Society of Anesthesiologists of Nepal (JSAN) has published its revised instruction to authors. The editorial board of JSAN has taken all possible steps to ensure the quality of the material published in the journal remains high and advise the authors to use the minimum set of items needed for a complete and clear description of the research methodology, findings of the study and discussion of the results. The new instructions to authors abide by the recommendations of the Equator Network and guide the authors to follow the checklists and guidelines to report their findings.

Using the reporting guidelines and checklists improves the accuracy, transparency, and completeness of the publications; and it will be easier for the readers to go through the article and judge the validity, reproducibility and reliability of the report. ${ }^{1,2,3}$ These guidelines attempt to improve quality through standardisation of reports. This article focuses on reporting guidelines that are relevant to JSAN and endorses them.

\section{Reporting randomised trials and the Consort Statement}

The authors are advised to follow the Consolidated Standards of Reporting Trials CONSORT statement to improve the quality of reporting of randomised trials. It is evidence-based and widely accepted set of tools to report trials. It was first published in 1996 and recently updated in 2010. It offers a standard way for authors to prepare reports of trial findings. It also facilitates complete and transparent reporting of the trials and aid critical judgment and analysis. The CONSORT Statement comprises a 25item checklist and a flow diagram. The checklist items help the authors on improving the paper on how the trial was designed, analysed, and interpreted. ${ }^{3}$ All the authors should develop the flow diagram as it displays the progress of all participants through the trial. JSAN strongly recommends that the flowchart is used in conjunction with the CONSORT Statement during submission.

Reporting observational studies and the STROBE statement STROBE stands for STrengthening the Reporting of OBservational studies in Epidemiology. The STROBE

How to cite this article: Sharma A, Aryal D. Guidelines for reporting health research: what authors should check before submitting an article. Journal of Society of Anesthesiologists of Nepal (JSAN) 2016;3(2):53-54. http://dx.doi.org/10.3126/ jsan.v3i2.15606

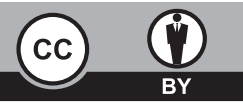

Corresponding Author: Apurb Sharma, MD

ORCID iD http://orcid.org/0000-0002-1655-5997,

Executive Editor, JSAN

Head, Department of Cardiac Anaesthesia, Shahid Gangalal National Heart Centre,

Bansbari, Kathmandu 44606, Nepal, Email: apurbsharma1976@gmail.com

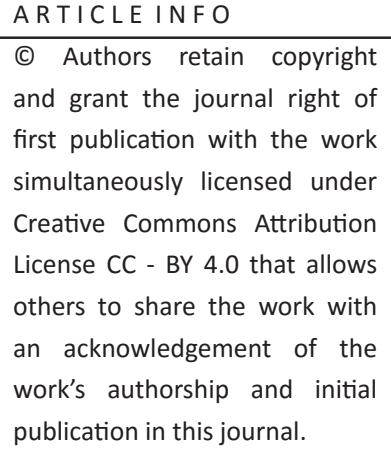

Journal of Society of Anesthesiologists of Nepal 
Statement was developed by the STROBE Initiative. It is an international collaboration of researchers, epidemiologists, statisticians, methodologists, and journal editors. The aim of the initiative is to assist authors when writing up analytical observational studies. It also supports editors and reviewers when considering observational studies for publication. It is extremely helpful for readers to critically appraise published studies. There are different checklists available for cohort studies, case-control studies and cross-sectional studies as well as there is a combined checklist for all these types of studies. The authors may choose the desired checklist as per their article type.

\section{CARE guidelines for Case Reports}

Case reports are published to express the adverse and beneficial effects of drugs or interventions, they may contribute to identifying new diseases, processes or unusual forms of common diseases, and they have been extensively used to present rare diseases. Case reports are common and almost one-third of all article types published in JSAN are case reports. In anaesthesia and intensive care, case reports are the main form of reporting mishaps. However, the quality and standard form of case reports are uneven from author to author. While reporting an interesting case, CARE guidelines provide a checklist of information to include in the reports for its standardisation. The CARE checklist contains 13 items to make the reports standard. The CARE website also contains case report writing templates and instruction to create an image of the timeline of events of the patients. JSAN urges all the authors to create the timeline for all relevant cases whenever applicable.

\section{The SQUIRE Guidelines for reporting quality improvement publication in healthcare}

SQUIRE 2.0 is the guideline for reporting quality improvement, for example, a Clinical audit published in JSAN. SQUIRE stands for Standards for QUality Improvement Reporting Excellence. The SQUIRE guidelines provide a framework for reporting novel information about how to improve healthcare. They are intended to improve the quality, safety, and value of healthcare.

Apart from these guidelines JSAN recommends the use of PRISMA Statement for reporting systematic reviews and meta-analyses evaluating health care interventions and the MOOSE guidelines for meta-analysis of observational studies.' Similarly, for reporting animal pre-clinical studies or in-vivo studies, the ARRIVE guidelines should be used. Other guidelines specific to different aspects of healthcare and their report are available at the Equator Network website.
The publication of papers after following these guidelines will help authors create standardised reports for editors, reviewers and readers. The Journal of Society of Anesthesiologists of Nepal endorses and recommends the use of these guidelines for every submission wherever applicable.

Conflict of Interests: Dr Sharma and Dr Aryal have nothing to disclose.

\section{References}

1. Equator network. Guidelines for reporting health research: How to promote their use in your journal [Internet]. Equator network [cited 12 August 2016]. Available from: http://www.equator-network.org/

2. Altman DG, Schulz KF, Moher D, Egger M, Davidoff F, Elbourne D, et al. The revised CONSORT statement for reporting randomized trials: explanation and elaboration. Ann Intern Med 2001;134:66394. http://dx.doi.org/10.7326/0003-4819-134-8-200104170-00012 [PMid:11304107]

3. Consort Group. Consolidated standards of reporting trials [Internet]. Consort Group [cited 12 August 2016]. Available from: http://www. consort-statement.org/

4. STROBE Initiative. Strengthening the Reporting of Observational Studies in Epidemiology (STROBE) [Internet]. Strobe initiative [cited August 12, 2016]. Available at: http://www.strobe-statement.org/

5. Gagnier JJ, Kienle G, Altman DG, Moher D, Sox H, Riley D, CARE Group. The CARE guidelines: consensus-based clinical case report guideline development. Journal of clinical epidemiology 2014;67:46-51. http://dx.doi.org/10.1016/j.jclinepi.2013.08.003 [PMid:24035173]

6. CARE Statement. Clinical case reporting guidelines [Internet]. CARE statement [cited August 12, 2016]. Available at: http://www.carestatement.org/

7. Moher D, Liberati A, Tetzlaff J, Altman DG, PRISMA Group. Preferred Reporting Items for Systematic Reviews and Meta-Analyses: The PRISMA Statement. PLOS Med 2009;6:e1000097. http:// dx.doi.org/10.1371/journal.pmed.1000097_[PMid:19621072] [PMCid:PMC2707599]

8. Stroup DF, Berlin JA, Morton SC, Olkin I, Williamson GD, Rennie D, et al. Meta-analysis of observational studies in epidemiology: a proposal for reporting. Meta-analysis Of Observational Studies in Epidemiology (MOOSE) group. JAMA 2000;283:2008-12. http:// dx.doi.org/10.1001/jama.283.15.2008 [PMid:10789670] 